University of Wollongong

Research Online

Australian Institute for Innovative Materials -

Papers

Australian Institute for Innovative Materials

$1-1-2016$

Correlation of impedance and effective electrode area of dextran sulfate doped PEDOT modified electrodes

\author{
Alexander R. Harris \\ University of Wollongong, alexh@uow.edu.au \\ Robyn C. Hutchinson \\ University of Wollongong, robynhut@uow.edu.au \\ Paul J. Molino \\ University of Wollongong, pmolino@uow.edu.au \\ Robert M. I Kapsa \\ University of Wollongong, robk@uow.edu.au \\ Graeme M. Clark \\ University of Melbourne, graeme.clark@latrobe.edu.au
}

See next page for additional authors

Follow this and additional works at: https://ro.uow.edu.au/aiimpapers

Part of the Engineering Commons, and the Physical Sciences and Mathematics Commons

Research Online is the open access institutional repository for the University of Wollongong. For further information contact the UOW Library: research-pubs@uow.edu.au 


\title{
Correlation of impedance and effective electrode area of dextran sulfate doped PEDOT modified electrodes
}

\author{
Abstract \\ The impedance of electrodes at $1 \mathrm{kHz}$ is typically reported to assess the signal-to-noise ratio of neural \\ recording electrodes. The impedance response of platinum electrodes modified by \\ poly-3,4-ethylenedioxythiophene doped with dextran sulfate has been examined. The modified electrodes \\ have lower impedance at low and intermediate frequencies compared to unmodified electrodes. The \\ impedance and phase angle at low frequencies is strongly correlated with the electrode area. The \\ geometric and linear diffusion charge densities of the modified electrodes are also dependent on the \\ electrode area and impedance at low frequencies. A 3 time constant equivalent circuit provided a better \\ fit to the impedance than a 2 time constant model. The decrease in impedance at low frequencies \\ indicates PEDOT-DS will be suitable for reducing the thermal noise and increasing the signal-to-noise ratio \\ for neural recording electrodes.
}

\section{Keywords}

correlation, impedance, effective, electrode, area, pedot, modified, dextran, doped, sulfate, electrodes

\section{Disciplines}

Engineering | Physical Sciences and Mathematics

\section{Publication Details}

Harris, A. R., Hutchinson, R., Molino, P. J., Kapsa, R. M. I., Clark, G. M., Paolini, A. G. \& Wallace, G. G. (2016). Correlation of impedance and effective electrode area of dextran sulfate doped PEDOT modified electrodes. Journal of the Electrochemical Society, 163 (7), H534-H540.

\section{Authors}

Alexander R. Harris, Robyn C. Hutchinson, Paul J. Molino, Robert M. I Kapsa, Graeme M. Clark, Antonio G. Paolini, and Gordon G. Wallace 
Correlation of Impedance and Effective Electrode Area of Dextran Sulphate Doped PEDOT Modified Electrodes

Alexander R. Harris ${ }^{1}$, Robyn Hutchinson ${ }^{2}$, Paul J. Molino ${ }^{2}$, Robert M.I. Kapsa ${ }^{2,3}$, Graeme M. Clark ${ }^{4}$, Antonio G. Paolini ${ }^{5,6}$, Gordon G. Wallace ${ }^{2}$

${ }^{1}$ HEARing CRC, Intelligent Polymer Research Institute, University of Wollongong, Wollongong, NSW, 2522, Australia

${ }^{2}$ ARC Centre of Excellence for Electromaterials Science, Intelligent Polymer Research Institute, University of Wollongong, NSW 2522, Australia.

${ }^{3}$ Department of Neurosciences, St Vincents Hospital, Melbourne and Department of Medicine University of Melbourne, Fitzroy, Victoria, 3065, Australia

${ }^{4}$ School of Engineering, University of Melbourne, Parkville, Victoria, 3010, Australia

${ }^{5}$ School of Health Science, RMIT University, Bundoora, Victoria, 3083, Australia

${ }^{6}$ School of Psychology and Public Health, La Trobe University, Bundoora, Victoria, 3086, Australia

Email: alexrharris@gmail.com 


\begin{abstract}
The impedance of electrodes at $1 \mathrm{kHz}$ is typically reported to assess the signal-to-noise ratio of neural recording electrodes. The impedance response of platinum electrodes modified by poly-3,4ethylenedioxythiophene doped with dextran sulfate has been examined. The modified electrodes have lower impedance at low and intermediate frequencies compared to unmodified electrodes. The impedance and phase angle at low frequencies is strongly correlated with the electrode area. The geometric and linear diffusion charge densities of the modified electrodes are also dependent on the electrode area and impedance at low frequencies. A 3 time constant equivalent circuit provided a better fit to the impedance than a 2 time constant model. The decrease in impedance at low frequencies indicates PEDOT-DS will be suitable for reducing the thermal noise and increasing the signal-to-noise ratio for neural recording electrodes.
\end{abstract}

\title{
Keywords
}

Electroactive polymer; Neural prosthesis; Surface analysis; Impedance Spectroscopy; Brain-machine interface 


\section{Introduction}

Neural electrodes are increasingly being used to understand how the brain functions; for the control of prosthetic devices; and relieving symptoms associated with several neural disorders including Parkinson's disease, epilepsy and chronic pain. Whilst recent developments in electrode technologies have delivered significant improvements in such devices, their performance is variable and progressively degenerative over chronic implantation time frames. Some improvements in short term performance have been achieved through modifying electrode geometry and chemical composition (1, 2), but a poor understanding of the electrode-tissue interface has prevented translation of these benefits to chronic implants.

Neural implants function as electrochemical transducers, converting electrical to ionic current. For neural recording applications, variations in ion concentration around excitable cells lead to changes in the local potential. This variation of potential can be measured by an electrode in close proximity to the target cells. To obtain a useful biological signal from the electrode, the measurement must have a large signal-to-noise ratio (SNR). The expected SNR of a neural electrode is typically assessed by measuring its impedance at $1 \mathrm{kHz}$. However, recently we provided an analytical solution to some common equivalent circuits that revealed that the electrode impedance at the intermediate frequency of $1 \mathrm{kHz}$ has a weak dependence on some electrode properties, including the electrode area. In contrast, the impedance at low frequencies was far more dependent on electrode area and may therefore be a better predictor of SNR (3). The impedance at intermediate frequencies was also greatly affected by electrode modification from the bare metal. Metal electrode modification with a doped conducting polymer changed the electrodes equivalent circuit, leading to a significant reduction of impedance at 1 kHz. However the difference in impedance at low and high frequencies between modified and unmodified electrodes was smaller and more variable. Comparison of different electrodes for neural implantation from a single impedance frequency, especially at $1 \mathrm{kHz}$, may not provide sufficient information to properly assess the electrodes effectiveness. Until a greater understanding of neural implant behaviour is achieved, measurement of the impedance over a wide frequency range is required.

The comparative biostability and biocompatibility of platinum to other electrode materials predicates its use in all commercial neural implants. However, encapsulation by scar tissue during chronic implantation reduces its recording SNR (4-6). Miniaturisation of the electrode may reduce the foreign body response to the implanted electrode (7); however this will increase its impedance, which will subsequently increase the electrodes SNR. Modification of the metal electrode surface to improve biocompatibility and reduce impedance has the potential to overcome these issues. A number of different materials, particularly doped conducting polymers, have been proposed for neural electrode modification, although to date, none have been stable over chronic implantation (8). 
Modification of electrodes with doped poly-3,4-ethylenedioxythiophene (PEDOT) has been shown to reduce electrode impedance at low to intermediate frequencies $(9,10)$. A number of studies have also demonstrated improvements in biocompatibility by varying the dopant ion, including a recent study using dextran sulfate (DS) as the dopant $(11,12)$. DS is a polysaccharide that acts as an antithrombotic. As a dopant ion trapped on the surface of a neural implant it has the potential to reduce the level of fouling and encapsulation on the electrode surface, leading to improved chronic performance. We recently investigated the voltammetric response of PEDOT-DS modified electrodes to gain an understanding of effective electrode area and charge density for neural stimulation (13). In this article we explore the impedance of these electrodes as a predictor of neural recording ability. The results are compared to PEDOT doped with para-toluene sulfonate (pTs) which we have previously recommended for acute neural recording applications (9).

\section{Experimental Section}

3,4-ethylenedioxythiophene (EDOT), dextran sulfate sodium salt (DS, average MW > 500,000), sodium para-toluene sulfonate ( $\mathrm{Na}_{2} \mathrm{pTs}$ ) (Sigma-Aldrich) and $99.0 \%$ di-sodium phosphate (Fluka) were used as received. Electrodes were coated as described previously $(13,14)$, briefly polymer coatings were deposited on $32,413 \mu \mathrm{m}^{2}$ nominal geometric area platinum electrodes (Neuronexus Technologies - A4x8-5mm-200-200-413). Conducting polymer deposition with various dopants were electrochemically deposited onto microelectrodes via a potentiostat (CH660D, CH Instruments) from solutions containing $10 \mathrm{mM}$ EDOT and $0.1 \mathrm{M} \mathrm{Na}_{2}$ pTs or $2 \mathrm{mg} \mathrm{mL}^{-1} \mathrm{DS}$ in deionised water. Deposition was performed in a three-electrode configuration using one electrode as working electrode, $\mathrm{Ag} / \mathrm{AgCl}$ $(3 \mathrm{M} \mathrm{NaCl})$ as reference electrode and $\mathrm{Pt}$ mesh as counter electrode. Solutions were degassed for 30 minutes with nitrogen before depositing the doped conducting polymers. All polymers were deposited at $1 \mathrm{~V}$ vs $\mathrm{Ag} / \mathrm{AgCl}$. PEDOT-DS was deposited for 4 growth times $(15,30,45$ or $60 \mathrm{~s})$, PEDOT-pTs was grown for $45 \mathrm{~s}$ as previously recommended $(9,15)$. 2 probes were coated with PEDOT-DS, 4 sites at each deposition time in a staggered format as described previously (9), leaving 12 uncoated platinum electrodes and 4 PEDOT-pTs coated electrodes as controls. Electrodeposition of conducting polymers produces homogeneous thin films $(16,17)$, further details on the morphology of the PEDOT-DS films are detailed in reference (13).

Electrode areas and charge density were determined optically and electrochemically as previously described (14). Images were collected on a BX61 optical microscope (Olympus) and the geometric area measured with ImageJ. A cross sectional SEM showed a uniform coating of approximately $15 \mathrm{~nm}$ thickness (Figure S1). Electrochemical analysis was performed in $0.3 \mathrm{M}$ phosphate buffer in deionised water and the electroactive areas determined by addition of $5 \mathrm{mM} \mathrm{Ru}\left(\mathrm{NH}_{3}\right)_{6}{ }^{3+}$. Test solutions weren't degassed to better represent conditions in vivo. A CHI660B potentiostat with CHI684 multiplexer (CH Instruments) were used to perform cyclic voltammetry and electrochemical impedance spectroscopy 
(EIS) at each of the individually addressable working electrode sites $(3,9,15)$. A 3 electrode configuration was used with a $\mathrm{Ag} / \mathrm{AgCl}(3 \mathrm{M} \mathrm{KCl})$ reference and $\mathrm{Pt}$ mesh counter electrode. Charge density measurements were performed via cyclic voltammetry over a potential window of 0.8 to $-0.8 \mathrm{~V}$ vs $\mathrm{Ag} / \mathrm{AgCl}$ at a scan rate of $100 \mathrm{mV} \mathrm{s}^{-1}$. Electroactive area measurements were collected from a potential window of 0 to $-0.5 \mathrm{~V}$ varying the scan rate from $10 \mathrm{mV} \mathrm{s}^{-1}$ to $1 \mathrm{~V} \mathrm{~s}^{-1}$. EIS was performed at $0 \mathrm{~V}$ with a $10 \mathrm{mV}$ amplitude over a frequency range of 10-100,000 Hz. Equivalent circuit fitting of the EIS data was performed with ZView.

\section{Results}

The voltammetric response of the PEDOT-DS (data not shown) was similar and increased with deposition time, indicating the charge movement in the film is consistent across all thicknesses used in this study. The impedance of uncoated platinum microelectrodes was typical for a series RC circuit (Figure 1) $(3,9)$. Modification of the electrode with doped PEDOT led to a different response that required a more complex equivalent circuit. PEDOT-DS modified electrodes showed an increase in impedance with decreasing frequency with a plateau region at intermediate frequencies (Figure 1d). The phase angle was around $-70^{\circ}$ at high frequencies, approached $0^{\circ}$ at intermediate frequencies and then became more negative at low frequencies (Figure 1e). The Nyquist plot displayed a semi-circle at high frequencies and approached a vertical response at low frequencies (Figure 1f). This impedance response at $0 \mathrm{~V}$ of PEDOT-DS modified electrodes in an electrolyte solution in the presence of dissolved oxygen is similar to PEDOT-pTs and PEDOT-PSS (3). PEDOT-DBSA modified electrodes tested in the same manner had a Nyquist plot where the low frequency region was much closer to $45^{\circ}$. This variation in shape of the Nyquist plot is most likely due to the measurement potential being at $0 \mathrm{~V}$ where a Faradaic process occurs on PEDOT-DBSA modified electrodes (10); the other modified electrodes do not show Faradaic peaks at this potential.

The impedance $(Z)$ is typically measured to assess thermal noise $\left(V_{r m s}^{\text {th }}\right)$ and the expected signal-to-noise ratio (SNR) of the electrode for electrophysiological recording, according to

$$
V_{r m s}^{t h}=\sqrt{k_{b} T Z \Delta f}
$$

where $k_{b}$ is Boltzmann's constant, $T$ is the absolute temperature and $\Delta f$ is the measuring bandwidth (18). Traditionally a frequency of $1 \mathrm{kHz}$ has been used for this assessment. At $1 \mathrm{kHz}$, increased deposition time of PEDOT-DS led to a slight decrease in impedance and less negative phase angle (Figure 1 and Table 1). Recently, we provided an analytical solution to the impedance of a simplified Randles circuit and normal Randles circuit which indicated that while impedance at $1 \mathrm{kHz}$ was affected by changing the equivalent circuit model, it provided little further information on the electrode properties (3). In contrast, low frequency impedance and phase angle were far more dependent on electrode area $(A)$ and may be a better predictor of electrophysiological performance. Clearly, at low frequencies, the 
impedance of PEDOT-DS modified electrodes is seen to decrease and the phase angle become less negative with longer deposition times (Figure 1 and Table 1).

While the analytical solution to the Randles circuit provides a relationship between electrode area and impedance, this equivalent circuit is not applicable to PEDOT-DS modified electrodes. Doped conducting polymers have been modelled previously as a series resistor, capacitor and finite length Warburg diffusion impedance (10). However, this type of model does not generate a time constant that would accommodate the semi-circle in the Nyquist plot seen in Figure 1. A more complex equivalent circuit is required for PEDOT-DS modified electrodes, beginning with a parallel capacitor and resistor element to fit the high frequency impedance. The time constant is also slightly depressed in height, so that the goodness of fit was still poor with a capacitor; in contrast a constant phase element $\left(\mathrm{CPE}_{1}\right)$ in parallel with a resistor $\left(\mathrm{R}_{2}\right)$ fit the high frequency impedance very well. A series finite length Warburg diffusion impedance $\left(\mathrm{W}_{1}\right)$ is required to fit the low frequency behaviour; and another series resistor $\left(\mathrm{R}_{1}\right)$ is included to account for solution resistance. Fitting of this 2 time constant equivalent circuit (Model 1 - Figure 2a) produced a reasonable average $\chi^{2}$ over 29 electrodes of $9.4 \times 10^{-4}$, however the fit to intermediate frequencies was poor $(\sim 50-1000 \mathrm{~Hz})$. Inclusion of another time constant consisting of a parallel capacitor $\left(\mathrm{C}_{1}\right)$ and resistor $\left(\mathrm{R}_{3}\right)$ led to a significant improvement in the goodness of fit in this region. Using this 3 time constant model (Model 2 - Figure 2b), the average $\chi^{2}$ over 30 electrodes was less than $8 \times 10^{-5}$, with an improving goodness of fit with increased PEDOT-DS deposition time. A comparison of the fit to PEDOT-DS deposited for $60 \mathrm{~s}$ using the two models is displayed in Figure 2c. Fitting with model 2 produces several fitting parameters including resistance $(R)$ values for $\mathrm{R}_{1}, \mathrm{R}_{2}$ and $\mathrm{R}_{3}$; a capacitance $(C)$ value for $\mathrm{C}_{1}$; an admittance $\left(Q_{0}\right)$ and power $(n)$ term for the constant phase element; and a diffusion resistance $\left(R_{\mathrm{D}}\right)$ and diffusional time constant $\left(\tau_{\mathrm{D}}\right)$ associated with the Warburg impedance. Table 2 provides the average fitted values from model 2 with different PEDOT-DS deposition times.

The real $(Z)$ and imaginary $\left(Z^{\prime \prime}\right)$ components of model 2 can be derived

$$
\begin{aligned}
& Z^{\prime}=R_{1}+R_{2}\left[\frac{1+a K_{2}}{K_{2}^{2}+2 a K_{2}+1}\right]+R_{3}\left[\frac{1}{1+\left(R_{3} \omega C_{1}\right)^{2}}\right]+R_{\mathrm{D}}\left[\left(\frac{1}{K_{4}}\right) \frac{\sinh K_{4}-\sin K_{4}}{\cosh K_{4}-\cos K_{4}}\right] \\
& Z^{\prime \prime}=R_{2}\left[\frac{-b K_{2}}{K_{2}^{2}+2 a K_{2}+1}\right]+R_{3}\left[\frac{R_{3} \omega C_{1}}{1+\left(R_{3} \omega C_{1}\right)^{2}}\right]+R_{\mathrm{D}}\left[\left(\frac{-1}{K_{4}}\right) \frac{\sinh K_{4}+\sin K_{4}}{\cosh K_{4}-\cos K_{4}}\right]
\end{aligned}
$$

where $\omega$ is the perturbation frequency and

$$
\begin{aligned}
& K_{2}=R_{2}\left(\omega Q_{0}\right)^{n} \\
& K_{4}=\sqrt{2 \omega \tau_{\mathrm{D}}}
\end{aligned}
$$




$$
\begin{aligned}
& a=\cos (90 n) \\
& b=\sin (90 n)
\end{aligned}
$$

This gives a total impedance

$$
\begin{gathered}
|Z|^{2}=Z^{\prime 2}+Z^{\prime \prime 2} \\
=\left(R_{1}+R_{2}\left[\frac{1+a K_{2}}{K_{2}^{2}+2 a K_{2}+1}\right]+R_{3}\left[\frac{1}{1+\left(R_{3} \omega C_{1}\right)^{2}}\right]+R_{\mathrm{D}}\left[\left(\frac{1}{K_{4}}\right) \frac{\sinh K_{4}-\sin K_{4}}{\cosh K_{4}-\cos K_{4}}\right]\right)^{2} \\
+\left(R_{2}\left[\frac{-b K_{2}}{K_{2}{ }^{2}+2 a K_{2}+1}\right]+R_{3}\left[\frac{R_{3} \omega C_{1}}{1+\left(R_{3} \omega C_{1}\right)^{2}}\right]+R_{\mathrm{D}}\left[\left(\frac{-1}{K_{4}}\right) \frac{\sinh K_{4}+\sin K_{4}}{\cosh K_{4}-\cos K_{4}}\right]\right)^{2}
\end{gathered}
$$

The phase angle $(\phi)$ can also be defined as

$$
\begin{gathered}
\phi=\tan ^{-1} \frac{Z^{\prime \prime}}{Z^{\prime}} \\
=\tan ^{-1}\left(\frac{R_{2}\left[\frac{-b K_{2}}{K_{2}^{2}+2 a K_{2}+1}\right]+R_{3}\left[\frac{R_{3} \omega C_{1}}{1+\left(R_{3} \omega C_{1}\right)^{2}}\right]+R_{\mathrm{D}}\left[\left(\frac{-1}{K_{4}}\right) \frac{\sinh K_{4}+\sin K_{4}}{\cosh K_{4}-\cos K_{4}}\right]}{\left.R_{1}+R_{2}\left[\frac{1+a K_{2}}{K_{2}^{2}+2 a K_{2}+1}\right]+R_{3}\left[\frac{1}{1+\left(R_{3} \omega C_{1}\right)^{2}}\right]+R_{\mathrm{D}}\left[\left(\frac{1}{K_{4}}\right) \frac{\sinh K_{4}-\sin K_{4}}{\cosh K_{4}-\cos K_{4}}\right]\right)}\right)
\end{gathered}
$$

The dependence on electrode area is achieved by substitution of capacitance according to

$$
C=\varepsilon \varepsilon_{0} A / d
$$

where $\varepsilon$ is the solution dielectric constant, $\varepsilon_{0}$ is the permittivity of free space and $d$ is the double layer thickness. At intermediate and high frequencies, the impedance and phase angle are independent of electrode area. At low frequencies, the relationship between total impedance and electrode area can be fitted to a trend line of the form $Z=\frac{a}{A^{2}}+\frac{b}{A}+c$ while the phase angle can be fitted to $\phi=a \tan ^{-1} \frac{b}{A}$

The geometric and electroactive electrode areas were determined by optical microscopy and reduction of $\mathrm{Ru}\left(\mathrm{NH}_{3}\right)_{6}{ }^{3+}$ respectively (13). Reduction of $\mathrm{Ru}\left(\mathrm{NH}_{3}\right)_{6}{ }^{3+}$ could be performed at slow and fast voltammetric scan rates to provide steady-state and linear diffusion electroactive areas. However the PEDOT-DS electrodes were too large to produce a steady-state response down to $10 \mathrm{mV} \mathrm{s}^{-1}$. Deposition of PEDOT-DS on metal electrodes increased the geometric and linear diffusion electroactive areas (13). In agreement with theory, plots of impedance at $1 \mathrm{kHz}$ versus geometric or linear diffusion electroactive area displayed no correlation (Figure $3 \mathrm{a}$ and $\mathrm{b}$ ). In contrast, very strong correlations of the form $Z=\frac{a}{A^{2}}+\frac{b}{A}+c$ were found between impedance at low frequencies (using $12 \mathrm{~Hz}$ as a representative frequency) and geometric and linear diffusion electroactive areas (Figure $3 \mathrm{c}$ and $\mathrm{d}$ ). The $\mathrm{R}^{2}$ was only 
slightly reduced by fitting a trend line of the form $Z=\frac{b}{A}+c$. A trend line of the form $\phi=a \tan ^{-1} \frac{b}{A}$ was fit to the phase angle at $12 \mathrm{~Hz}$ versus geometric and linear diffusion electroactive areas giving good $\mathrm{R}^{2}$ values, which were very similar to a linear trend line (Figure 4 ).

After fitting the model 2 equivalent circuit, a number of parameters are obtained (table 2). Each of these can also be plotted against the geometric and linear diffusion electroactive areas (Figure 5). Both $Q_{0}$ and $R_{\mathrm{D}}$ show little correlation with electrode area; $C_{1}$ and $\tau_{\mathrm{D}}$ displayed a linear trend with electrode area; and $R_{3}$ had a correlation with electrode area of the form $R=\frac{a}{A^{2}}+\frac{b}{A}+c$.

The charge density of the electrode is also typically measured to assess the ability of an electrode to stimulate neurons. An electrode with a larger charge density is able to deliver sufficient charge to excite a neuron from a smaller area, allowing the electrode size to be reduced, subsequently increasing the devices biocompatibility and potential ability to stimulate individual neurons. The charge density $(\mathrm{mC}$ $\mathrm{cm}^{-2}$ ) is typically measured from the charge passed during a voltammogram and the nominal electrode area. A greater understanding of charge density was obtained by measuring the geometric and electroactive areas to provide geometric and electroactive charge densities (14). Plots of impedance at $1 \mathrm{kHz}$ versus geometric and linear diffusion charge density displayed no correlation, however all PEDOT-DS modified electrodes possessed larger geometric charge densities and smaller linear diffusion charge densities than unmodified electrodes (Figure 6a and b). Plots of impedance at $12 \mathrm{~Hz}$ versus charge density also show all PEDOT-DS modified electrodes possessed larger geometric charge densities and smaller linear diffusion charge densities than unmodified electrodes (Figure $6 \mathrm{c}$ and d). It was found that the charge density of the PEDOT-DS modified electrodes were correlated to electrode area (13). In further agreement with this result, at small charge densities, the impedance at $12 \mathrm{~Hz}$ also displayed a trend of increasing charge density with decreasing impedance.

\section{Discussion}

Modification of neural electrodes with PEDOT-DS led to an increase in effective electrode area and charge density (13). This may result in improved electrode properties for neural stimulation applications. For neural recording, the electrode impedance is usually measured, as the impedance affects the thermal noise (equation 1) and subsequently the signal-to-noise ratio. Bare metal electrodes display a linear impedance versus frequency response which can be modelled as a simple RC network. This results in an inverse dependence of electrode area with impedance at all frequencies. The impedance of modified electrodes is more complex than bare metal electrodes, the non-linear PEDOTDS response requiring a 3 time constant equivalent circuit to achieve a good fit. When determining the potential thermal noise and SNR from more complex equivalent circuits, choice of measurement 
frequency will affect the SNR prediction. Typically SNR for neural electrodes has been assessed at 1 $\mathrm{kHz}$, and it is clear that by simply modifying the electrodes to conform to a number of different equivalent circuits, a reduction in impedance occurs at this intermediate frequency.

It currently isn't known which impedance frequencies lead to a reduction in thermal noise for neural recording. If the impedance at $1 \mathrm{kHz}$ is important, than many forms of electrode modification and their associated equivalent circuits can be utilised to reduce the electrode impedance. However, it is likely that the thermal noise and SNR is dependent on the electrode area. For an RC circuit, the impedance at all frequencies is dependent on electrode area, so that prediction of SNR could be achieved by measuring the impedance at any frequency. On electrodes with more complex equivalent circuits, the impedance at $1 \mathrm{kHz}$ is not dependent on electrode area. In this case, measurement of impedance at 1 $\mathrm{kHz}$ may be a poor predictor of SNR. In contrast, the impedance and phase angle at low frequencies is highly dependent on electrode area.

In contrast to previous reports of electrochemical impedance spectroscopy of doped conducting polymers, where a 3 element equivalent circuit has been used $(10,19)$, an equivalent circuit producing 3 time constants was required to fit the impedance of PEDOT-DS modified electrodes in a solution containing oxygen. The first element required accounts for the solution resistance $\left(\mathrm{R}_{1}\right)$; the interface between the conducting polymer and electrolyte solution double layer includes a parallel constant phase element $\left(\mathrm{CPE}_{1}\right)$ and polarisation resistance $\left(\mathrm{R}_{3}\right)$, the use of a constant phase element rather than a capacitor is usually explained as surface roughness or inhomogeneity in current distribution at the electrode surface; a finite length Warburg diffusion impedance $\left(\mathrm{W}_{1}\right)$ is required to account for the conducting polymers Faradaic process; finally a parallel capacitor $\left(\mathrm{C}_{1}\right)$ and resistor $\left(\mathrm{R}_{3}\right)$ element is included to achieve a good fit in the intermediate frequency region. The appearance of this final time constant may be due to the presence of oxygen in the solution, generating a charge transfer process (10). However attempts to fit a circuit including a second Warburg impedance element in place of $C_{1}$ and $R_{3}$ gave a very poor fit, suggesting this process isn't associated with oxygen. There were correlations between the electrode area and fitting parameters $C_{1}$ and $R_{3}$. Therefore it is more likely this process is due to dispersion in the electrode surface properties, which is dependent on the PEDOT-DS deposition time. The increased deposition time subsequently affects the electrode geometry and potentially its surface chemical structure. Further work is required to gain a greater understanding of this behaviour. The fitted parameters for $\mathrm{R}_{1}, \mathrm{C}_{1}$ and $\tau_{\mathrm{D}}$ with model 2 are in very good agreement with corresponding elements from a 3 parameter model used for fitting PEDOT-PSS on gold neural electrodes (19).

After fitting a suitable equivalent circuit to the impedance, various fitting parameters also display dependence on electrode area. If the electrode area is an important property for determining thermal noise and SNR, then these parameters may become useful predictors of electrophysiological response. 
However care must be taken to ensure that the equivalent circuit is a valid physical model. Furthermore, Figure 6 highlights that the relationship between each parameter and electrode area is not a simple linear dependence. The correlation between $\mathrm{C}_{1}$ and electrode area was very strong, particularly the linear diffusion electroactive area. In contrast, the correlation of $R_{3}$ and $\tau_{\mathrm{D}}$ with electrode area were not as strong (compare Figures 3 and 5). The comparison of the impedance at $12 \mathrm{~Hz}$ and $1 \mathrm{kHz}$ as well as each fitting parameter to neural recording data (9) will provide great insight into the key electrode properties for determining their SNR.

Regardless of the specific choice of equivalent circuit, PEDOT-DS modification reduced the electrode impedance at all low to intermediate frequencies. The use of this doped conducting polymer should therefore reduce the thermal noise and improve the signal-to-noise ratio of neural recording electrodes. As DS is an antithrombotic, it's inclusion in the electrode coating may also result in improved biocompatibility, resulting in more stable chronic performance.

PEDOT-DS modification also produced larger optical charge densities and lower linear diffusion charge densities than unmodified electrodes (13). This suggests that a greater, more homogenous charge can be delivered from the electrode surface compared to PEDOT and PPy doped with sulphate, para-toluene sulfonate (pTs), poly(styrenesulfonate) (PSS) and dodecylbenzenesulfonate (DBSA). This could produce more consistent neural stimulation behaviour. It was also found that the charge density was dependent on the geometric and linear diffusion electroactive area (13). Correlation of impedance at low frequencies with geometric and linear diffusion charge density further highlights the relationship between effective electrode area and charge density. The charge density of a material is generally assumed to be independent of its geometry. The dependence of charge density on effective electrode area, and hence impedance at low frequencies, has important implications for the reporting of single charge density values for a material. An ability to modify the charge density and impedance of a material by altering its effective area allows significant improvement in the performance of both neural stimulating and recording electrodes.

Electrodes coated with PEDOT-DS displayed similar coefficients of variation in electrode area compared to PEDOT-pTs, but larger coefficients of variation in impedance, phase angle and some charge density measurements. It is unclear as to whether this will lead to an increase in error of electrophysiological performance. Systematic measurement of the electrophysiological performance of these PEDOT-DS modified electrodes will provide important information on the impact of electrochemical variation on neuron-electrode interaction. Overall, the electrochemical characteristics of PEDOT-DS modified electrodes and biocompatibility evident in the adhesion of extracellular matrix proteins and PC12 neuronal cells (11) suggest that PEDOT-DS may be of significant value for improving electrode function in vivo, in particular by reducing SNR. 


\section{Conclusion}

PEDOT-DS modification of microelectrodes can be fit with a 3 time constant equivalent circuit. The impedance at low and intermediate frequencies is reduced compared to unmodified electrodes. The electrode area controls the impedance and phase angle at low frequencies. The geometric and linear diffusion charge densities are also dependant on electrode area. The low impedance of PEDOT-DS modified electrodes indicates they will reduce the thermal noise and increase the signal-to-noise ratio of neural recording electrodes. The large charge density of the electrodes is also favourable for neural stimulating electrodes.

\section{Acknowledgements}

Funding from the Australian Research Council Centre of Excellence Scheme (Project Numbers CE0561616 and CE140100012) are gratefully acknowledged. GGW is also grateful to the ARC for support under the Australian Laureate Fellowship scheme (FL110100196). The authors acknowledge the support of the Australian National Fabrication Facility (ANFF).

\section{References}

1. X. Cui, J. F. Hetke, J. A. Wiler, D. J. Anderson and D. C. Martin, Sensors and Actuators A: Physical, 93, 8 (2001).

2. D. J. Edell, V. V. Toi, V. M. McNeil and L. D. Clark, Biomedical Engineering, IEEE Transactions on, 39, 635 (1992).

3. A. R. Harris, P. J. Molino, R. M. I. Kapsa, G. M. Clark, A. G. Paolini and G. G. Wallace, Analyst, 140, 3164 (2015).

4. W. Grill and J. Thomas Mortimer, Annals of Biomedical Engineering, 22, 23 (1994).

5. P. J. Rousche and R. A. Normann, Journal of Neuroscience Methods, 82, 1 (1998).

6. S. Suner, M. R. Fellows, C. Vargas-Irwin, G. K. Nakata and J. P. Donoghue, Neural Systems and Rehabilitation Engineering, IEEE Transactions on, 13, 524 (2005).

7. T. D. Yoshida Kozai, N. B. Langhals, P. R. Patel, X. Deng, H. Zhang, K. L. Smith, J. Lahann, N. A. Kotov and D. R. Kipke, Nat Mater, 11 (2012).

8. A. F. Quigley, J. M. Razal, M. Kita, R. Jalili, A. Gelmi, A. Penington, R. Ovalle-Robles, R. H. Baughman, G. M. Clark, G. G. Wallace and R. M. I. Kapsa, Advanced Healthcare Materials, 1, 801 (2012).

9. A. R. Harris, S. J. Morgan, J. Chen, R. M. I. Kapsa, G. G. Wallace and A. G. Paolini, Journal of Neural Engineering, 10, 016004 (2013).

10. J. Bobacka, A. Lewenstam and A. Ivaska, Journal of Electroanalytical Chemistry, 489, 17 (2000).

11. P. J. Molino, Z. Yue, B. Zhang, A. Tibbens, X. Liu, R. M. I. Kapsa, M. J. Higgins and G. G. Wallace, Advanced Materials Interface, 1, 1300122 (2014).

12. A. Gelmi, M. K. Ljunggren, M. Rafat and E. W. H. Jager, Journal of Materials Chemistry B, 2, 3860 (2014).

13. A. R. Harris, P. J. Molino, R. M. I. Kapsa, G. M. Clark, A. G. Paolini and G. G. Wallace, Synthetic Metals (Submitted).

14. A. R. Harris, P. J. Molino, R. M. I. Kapsa, G. M. Clark, A. G. Paolini and G. G. Wallace, Analytical Chemistry, 87, 738 (2015). 
15. A. R. Harris, S. J. Morgan, G. G. Wallace and A. G. Paolini, Journal of Visualized Experiments, e51084 (2014).

16. E. Tamburri, S. Orlanducci, F. Toschi, M. L. Terranova and D. Passeri, Synthetic Metals, 159, 406 (2009).

17. V. Castagnola, C. Bayon, E. Descamps and C. Bergaud, Synthetic Metals, 189, 7 (2014).

18. G. Baranauskas, E. Maggiolini, E. Castagnola, A. Ansaldo, A. Mazzoni, G. N. Angotzi, A. Vato, D. Ricci, S. Panzeri and L. Fadiga, Journal of Neural Engineering, 8, 066013 (2011).

19. X. Cui and D. C. Martin, Sensors and Actuators B: Chemical, 89, 92 (2003). 


\section{Figure Captions}

Figure 1: Typical impedance response of electrodes in $0.3 \mathrm{M} \mathrm{Na}_{2} \mathrm{HPO}_{4}$ at $0 \mathrm{~V}$ and $10 \mathrm{mV}$ amplitude (ac) uncoated and PEDOT-pTs deposited for $45 \mathrm{~s}$, and (d-f) PEDOT-DS at varying deposition times. (f) data points with calculated curve using model 2 .

Figure 2: (a) Model 1 and (b) model 2 equivalent circuits used for PEDOT-DS modified electrodes in the presence of oxygen, $R_{1}$ is the solution resistance, $C P E_{1}$ is the constant phase element, $R_{2}$ and $R_{3}$ are resistors, $\mathrm{C}_{1}$ is a capacitor and $\mathrm{W}_{1}$ is a finite length Warburg diffusion impedance. (c) Comparison of model 1 and 2 fitting to PEDOT-DS deposited for $60 \mathrm{~s}$.

Figure 3: Comparison of impedance at ( $\mathrm{a}$ and b) $1 \mathrm{kHz}$ and (c and d) $12 \mathrm{~Hz}$ of PEDOT-DS modified electrodes measured in $0.3 \mathrm{M} \mathrm{Na}_{2} \mathrm{HPO}_{4}$ at $0 \mathrm{~V}$ and $10 \mathrm{mV}$ amplitude with electrode area measured (a and c) optically or ( $\mathrm{b}$ and d) by electrochemical reduction of $5 \mathrm{mM} \mathrm{Ru}\left(\mathrm{NH}_{3}\right)_{6}{ }^{3+}$ at linear diffusion. (a and b) The fitted trendline is linear, (c and d) the trendline is of the form $Z=\frac{a}{A^{2}}+\frac{b}{A}+c$.

Figure 4: Comparison of phase angle at $12 \mathrm{~Hz}$ of PEDOT-DS modified electrodes measured in $0.3 \mathrm{M}$ $\mathrm{Na}_{2} \mathrm{HPO}_{4}$ at $0 \mathrm{~V}$ and $10 \mathrm{mV}$ amplitude with electrode area measured (a) optically or (b) by electrochemical reduction of $5 \mathrm{mM} \mathrm{Ru}\left(\mathrm{NH}_{3}\right)_{6}{ }^{3+}$ at linear diffusion. The fitted trendline is linear.

Figure 5: Comparison of model values versus geometric area and linear diffusion electroactive area.

The fitted trendlines of $Q_{0}, C_{1}, R_{\mathrm{D}}$ and $\tau_{\mathrm{D}}$ are linear, the trendline of $R_{3}$ is of the form $R=\frac{a}{A^{2}}+\frac{b}{A}+c$

Figure 6: Comparison of impedance at ( $\mathrm{a}$ and b) $1 \mathrm{kHz}$ and (c and d) $12 \mathrm{~Hz}$ of PEDOT-DS modified electrodes measured in $0.3 \mathrm{M} \mathrm{Na}_{2} \mathrm{HPO}_{4}$ at $0 \mathrm{~V}$ and $10 \mathrm{mV}$ amplitude versus charge density with an electrode area measured ( $\mathrm{a}$ and $\mathrm{c}$ ) optically or (b and d) by electrochemical reduction of $5 \mathrm{mM}$ $\mathrm{Ru}\left(\mathrm{NH}_{3}\right)_{6}{ }^{3+}$ at linear diffusion. 


\section{Figures}

Table 1. Average, standard deviation and coefficient of variation of impedance (kOhm).

\begin{tabular}{lccccccccc}
\hline \multirow{2}{*}{ Polymer coating } & \multicolumn{3}{c}{ Impedance $1 \mathrm{kHz}$} & \multicolumn{3}{c}{ Impedance 12 Hz } & \multicolumn{3}{c}{ Phase Angle 12 Hz } \\
\cline { 2 - 11 } & Ave & SD & CV & Ave & SD & CV & Ave & SD & CV \\
\hline 15s PEDOT-DS & 26.8 & 1.4 & 0.05 & 237.5 & 41.6 & 0.17 & -81.1 & 5.0 & 0.06 \\
30s PEDOT-DS & 24.9 & 4.1 & 0.16 & 98.8 & 15.6 & 0.16 & -70.9 & 9.5 & 0.13 \\
45s PEDOT-DS & 23.3 & 3.7 & 0.16 & 62.5 & 7.7 & 0.12 & -63.5 & 6.1 & 0.10 \\
60s PEDOT-DS & 23.5 & 3.2 & 0.13 & 45.3 & 5.7 & 0.12 & -49.7 & 9.1 & 0.18 \\
45s PEDOT-pTs & 29.7 & 1.8 & 0.06 & 250.7 & 17.8 & 0.07 & -79.6 & 2.3 & 0.03 \\
Uncoated & 307.9 & 22.5 & 0.07 & 23693.9 & 8441.2 & 0.36 & -91.8 & 27.4 & 0.30 \\
\hline
\end{tabular}

Table 2. Average electrochemical impedance parameters from fitting model 2.

\begin{tabular}{lcccccccc}
\hline Polymer coating & $R_{1} / \Omega$ & $\begin{array}{c}Q_{0} / 10^{-9} \\
\mathrm{~S} \mathrm{~s} \mathrm{~s}^{1 / 2}\end{array}$ & $n$ & $R_{2} / \mathrm{k} \Omega$ & $C_{1} / \mathrm{nF}$ & $\begin{array}{c}R_{3} \\
/ \mathrm{M} \Omega\end{array}$ & $\begin{array}{c}R_{\mathrm{D}} \\
/ \mathrm{k} \Omega\end{array}$ & $\begin{array}{c}\tau_{\mathrm{D}} \\
/ \mathrm{ms}\end{array}$ \\
\hline 15s PEDOT-DS & 284 & 0.67 & 0.95 & 24.2 & 91.1 & 4.86 & 8.34 & 1.27 \\
30s PEDOT-DS & 345 & 0.63 & 0.95 & 23.0 & 224.0 & 1.93 & 10.8 & 4.58 \\
45s PEDOT-DS & 357 & 0.73 & 0.94 & 22.0 & 330.1 & 2.05 & 13.1 & 11.84 \\
60s PEDOT-DS & 395 & 0.66 & 0.95 & 22.4 & 548.3 & 0.80 & 15.9 & 19.41 \\
\hline
\end{tabular}

a

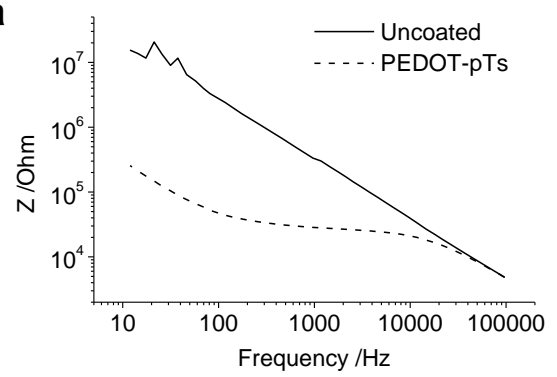

d

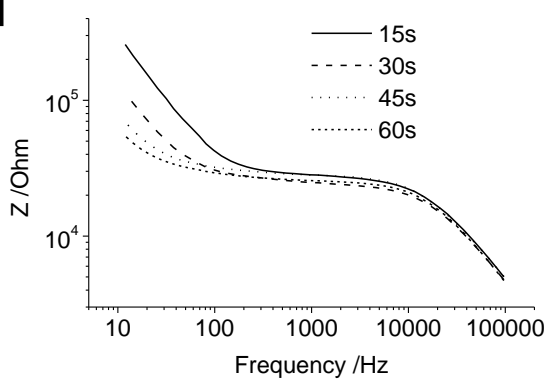

Figure 1 b

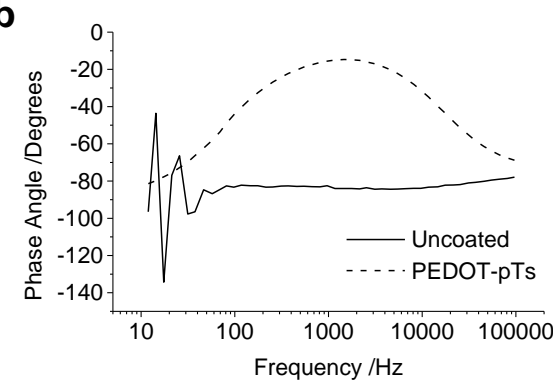

e

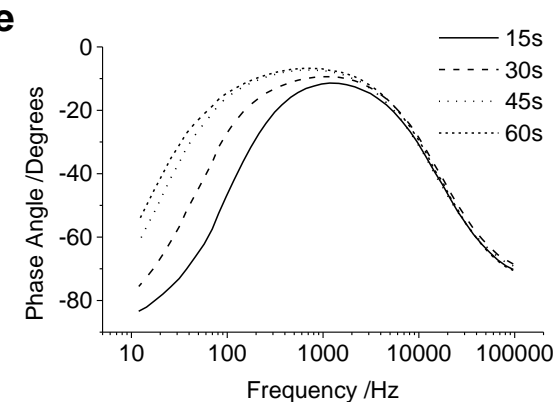

C

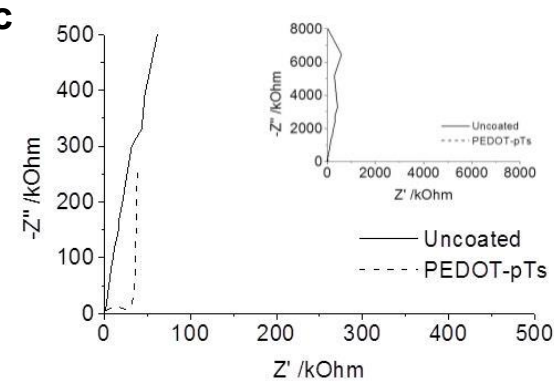

f

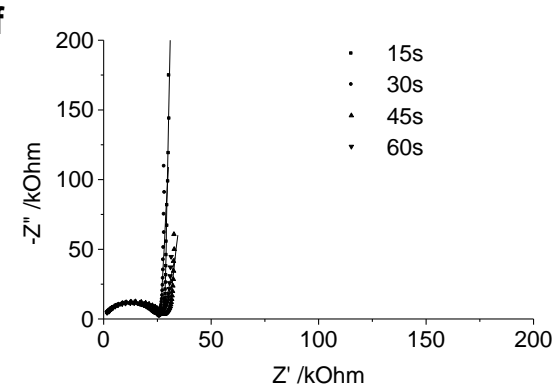


a

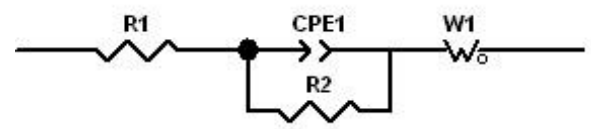

b

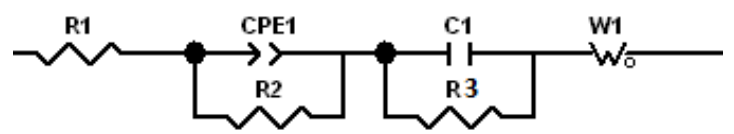

Figure 2
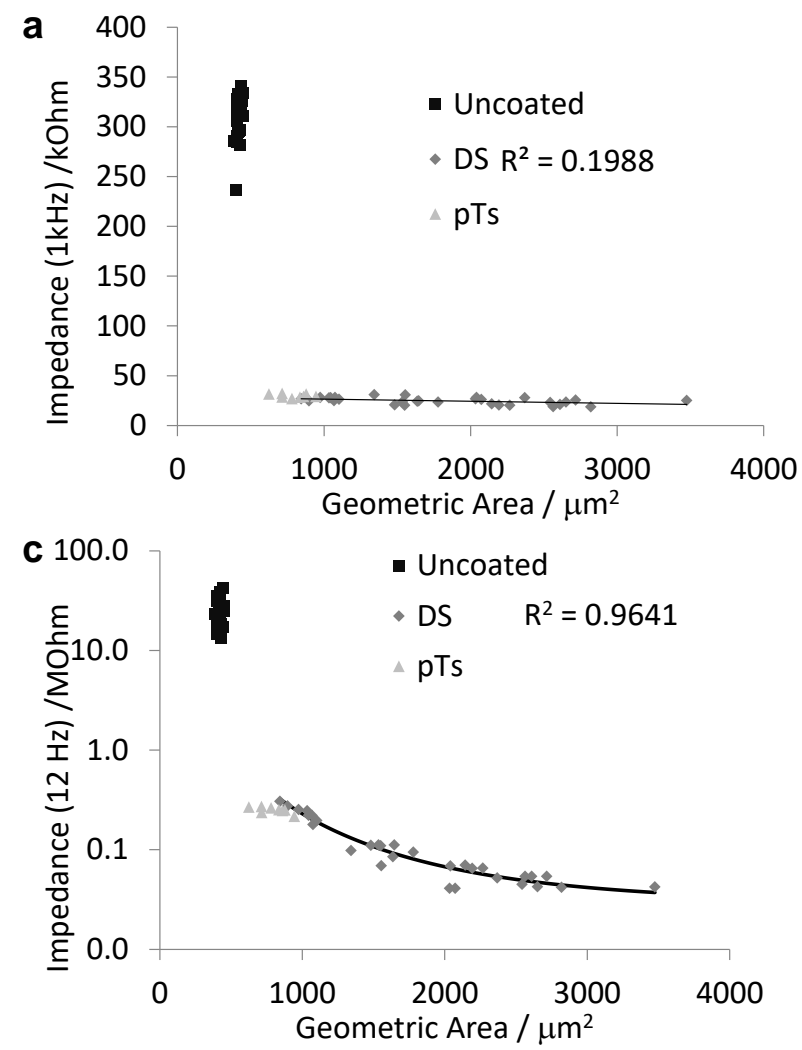

Figure 3
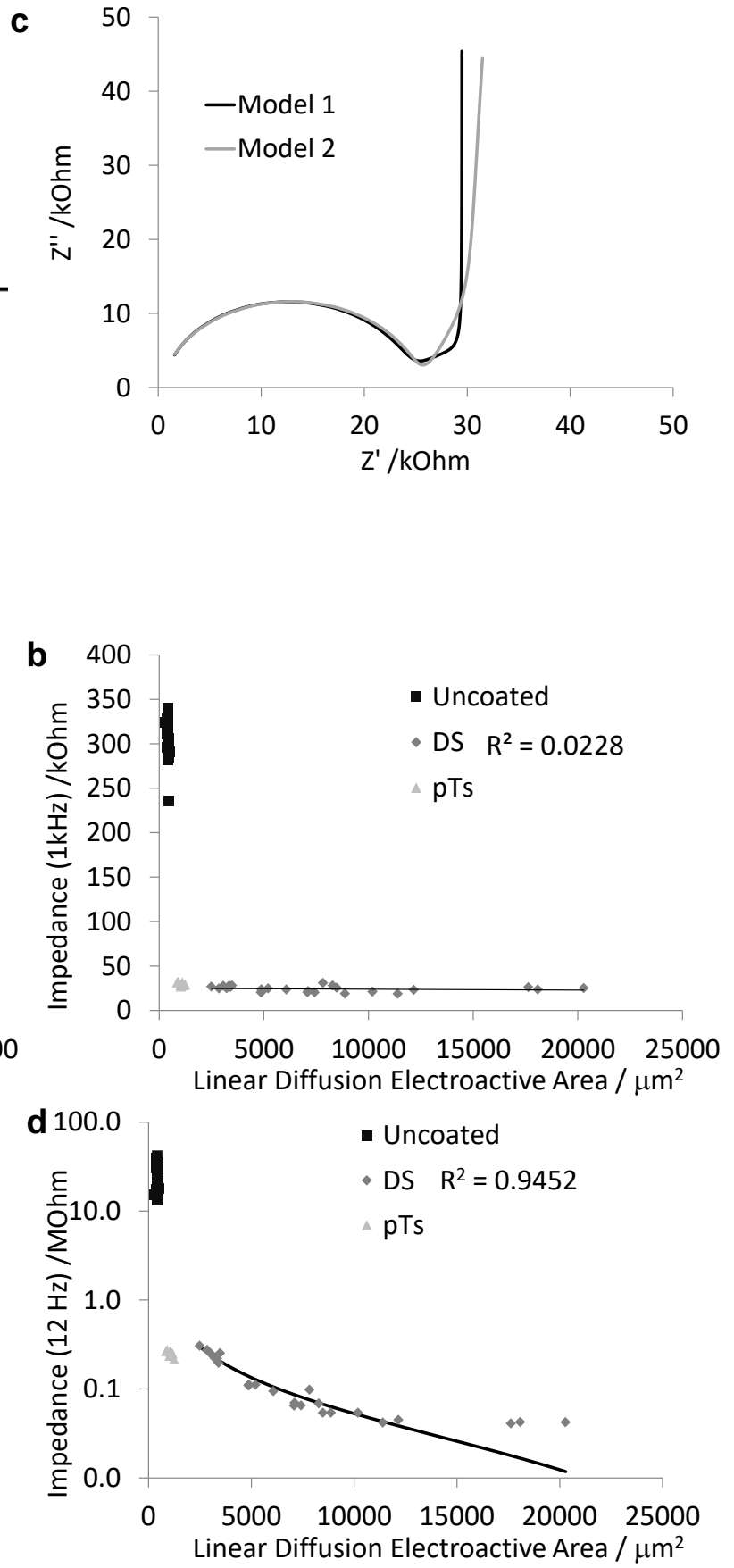

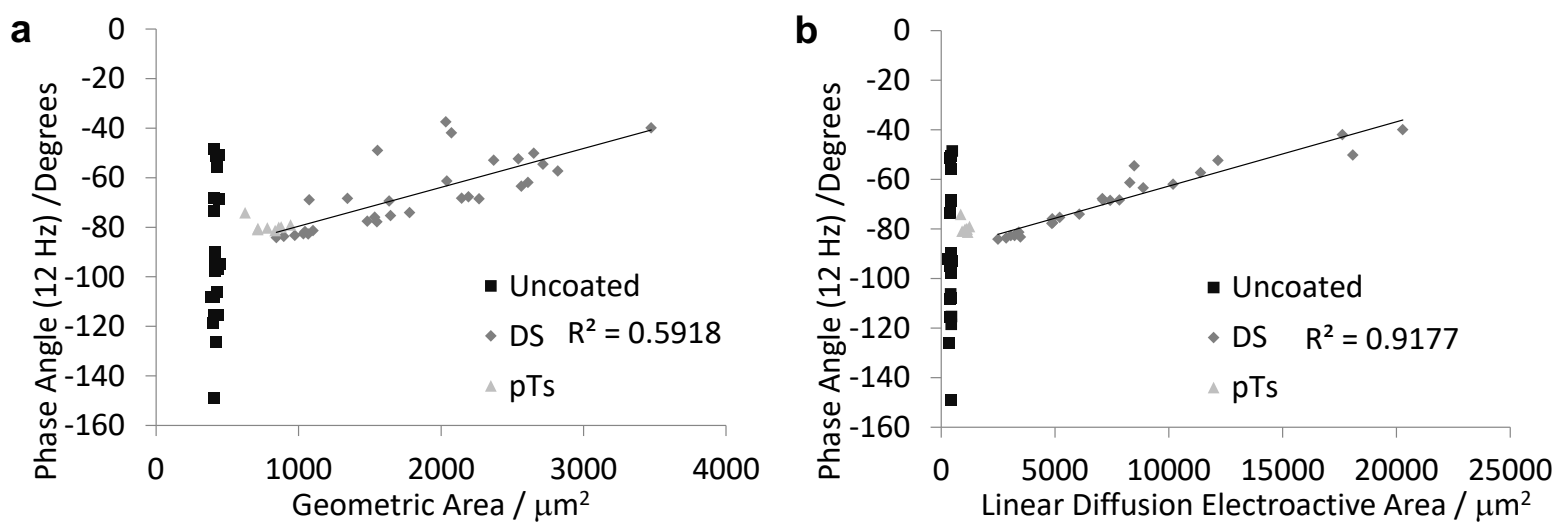

Figure 4
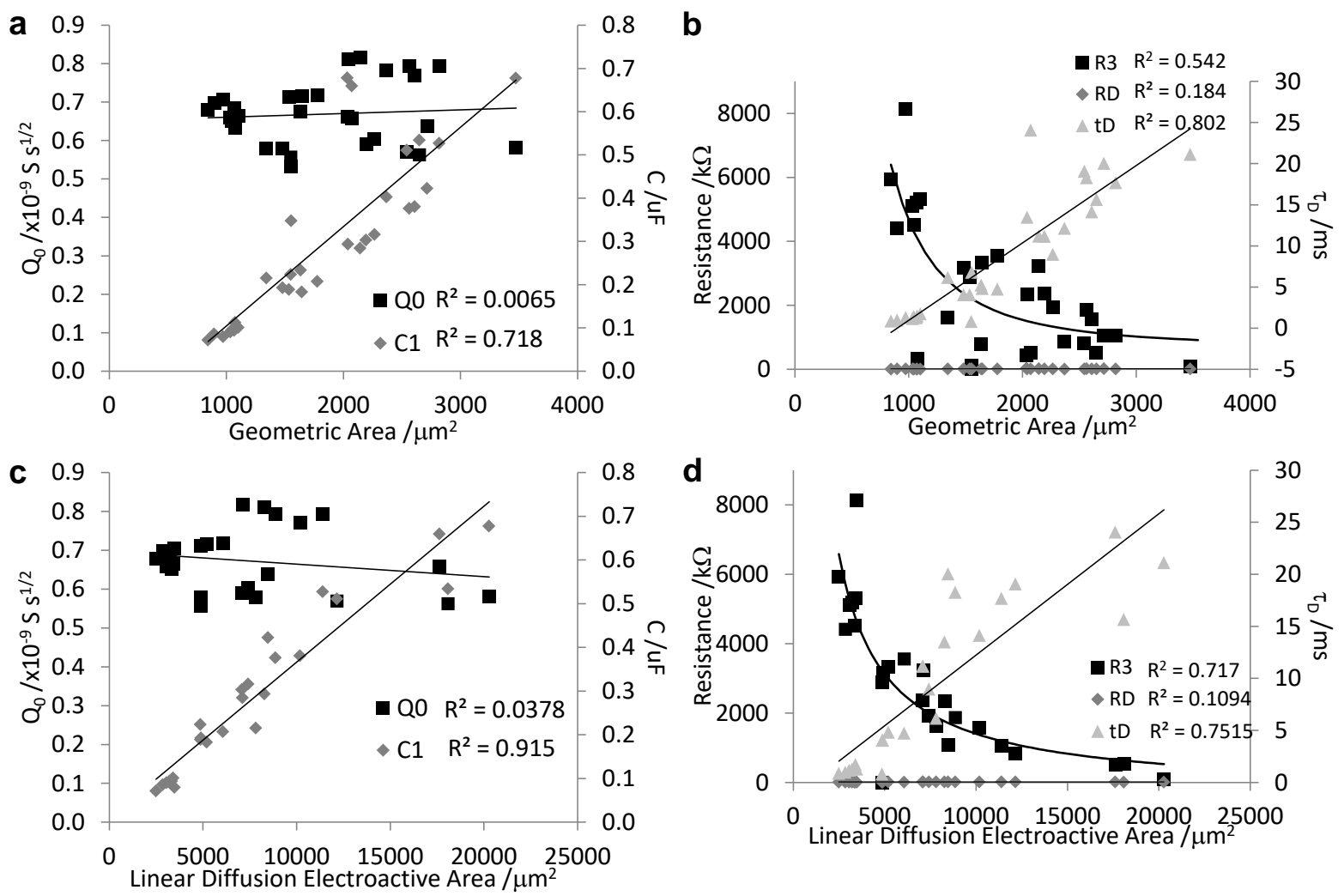

Figure 5 

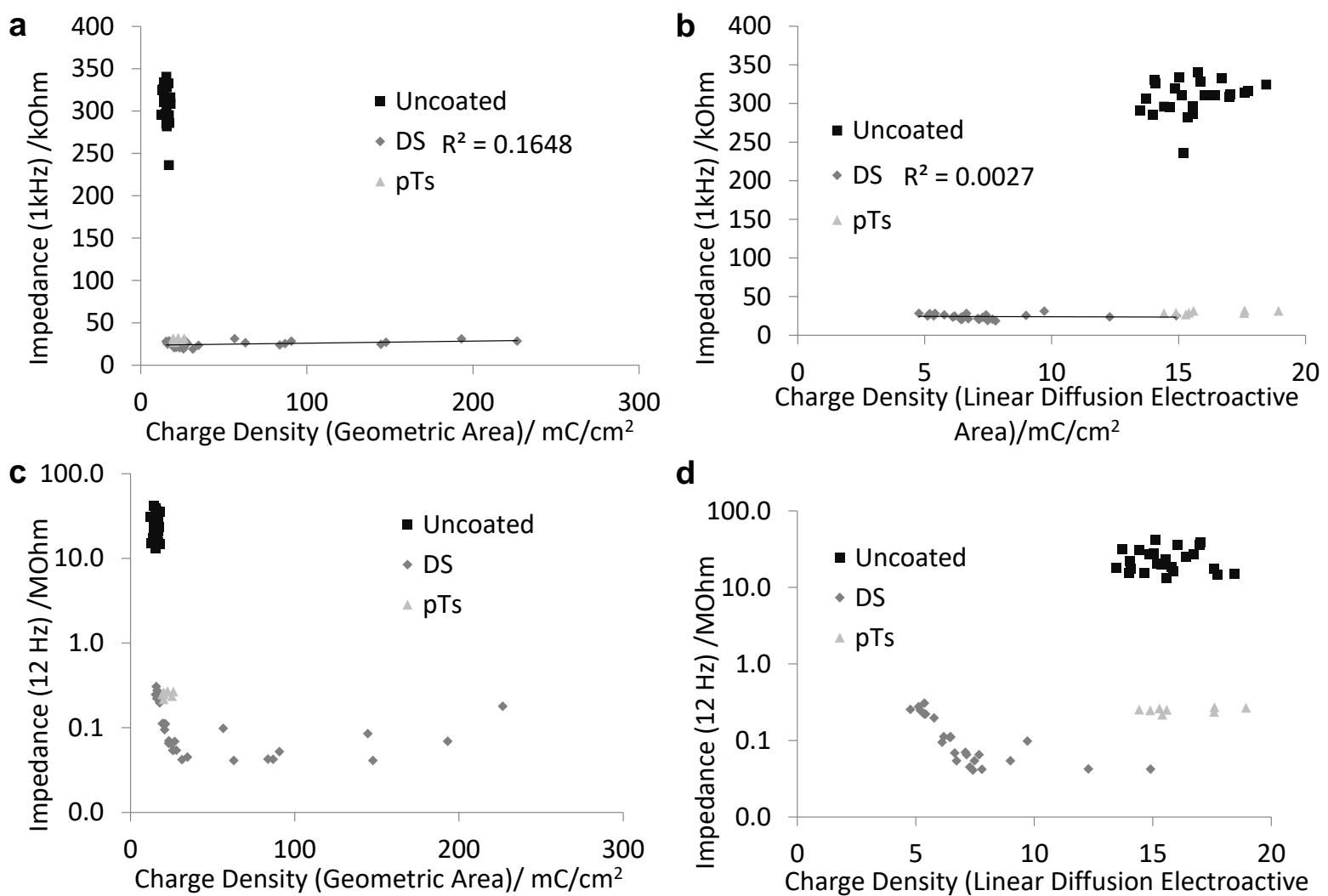

d Area) $/ \mathrm{mC} / \mathrm{cm}^{2}$

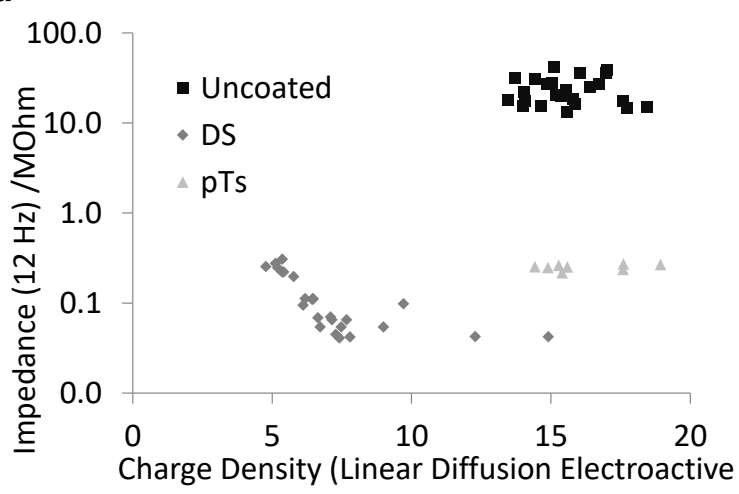
Area) $/ \mathrm{mC} / \mathrm{cm}^{2}$

Figure 6 


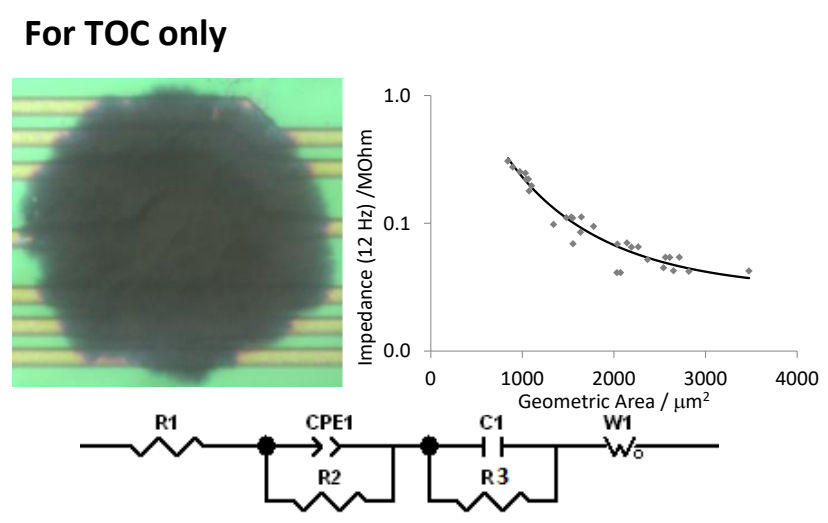

